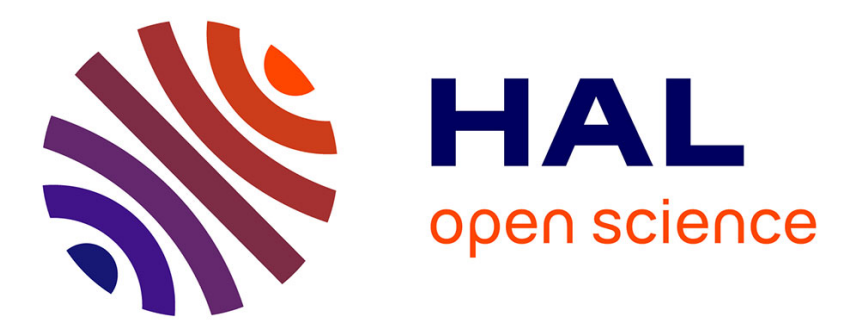

\title{
Time derivatives of screws with applications to dynamics and stiffness
}

Harvey Lipkin

\section{To cite this version:}

Harvey Lipkin. Time derivatives of screws with applications to dynamics and stiffness. Mechanism and Machine Theory, 2005, 40 (3), 10.1016/j.mechmachtheory.2003.07.002 . hal-01371131

\section{HAL Id: hal-01371131 \\ https://hal.science/hal-01371131}

Submitted on 24 Sep 2016

HAL is a multi-disciplinary open access archive for the deposit and dissemination of scientific research documents, whether they are published or not. The documents may come from teaching and research institutions in France or abroad, or from public or private research centers.
L'archive ouverte pluridisciplinaire HAL, est destinée au dépôt et à la diffusion de documents scientifiques de niveau recherche, publiés ou non, émanant des établissements d'enseignement et de recherche français ou étrangers, des laboratoires publics ou privés. 


\title{
Time derivatives of screws with applications to dynamics and stiffness
}

\author{
Harvey Lipkin \\ George W. Woodruff School of Mechanical Engineering, Georgia Institute of Technology, Atlanta, \\ GA 30332 0405, United States \\ E mail address: harvey.lipkin@me.gatech.edu
}

\begin{abstract}
Screw quantities provide geometric insight into three-dimensional mechanics modeled by rigid bodies and lumped parameters. Four distinct cases of time differentiation are examined by combining fixed and moving body derivatives (fundamental to rigid body mechanics) with material and local derivatives (fun-damental to continuum mechanics). Three combinations always yield another screw quantity while the most common, the material derivative with respect to the fixed body, does not. Two fundamental formulations are examined with this last derivative, Euler's Laws and the gravitational loading of an elastic system. By coincidence, the formulations appear screw-like when they are expressed at the center-of-mass but, in contrast to actual screw formulations, they do not retain invariant forms when expressed at arbitrary points.
\end{abstract}

\section{Introduction}

Screw quantities are used for three-dimensional mechanics that are treatable as rigid body systems with lumped-parameter models. They impart direct geometrical insight into physical phenomena. Ball [1] used screws to geometrically investigate the kinematics and dynamics of rigid 
body systems, and a symposium in his honor well illustrates the breadth and variety of modern screw theory applications [14]. Comprehensive treatments in kinematics and mechanism theory are presented by Bottema and Roth [2], Hunt [11], and Phillips [18].

Although it would appear that the time derivative of screw quantity should be well understood, that is not the case. There is active discussion in the literature of: the general topic by [21,25,29]; applications to acceleration analysis by [22,8], with historical background in [20]; applications to stiffness by [9,5,10,26,24]; and applications to dynamics by $[15,7,13,4,17,27]$. A growing trend is to use differential geometry, and in particular, Lie algebras, such as presented in $[12,23]$.

Two important classical works are Brand [3] and von Mises [28] who use motor representations for the derivative of a screw. Interestingly, the Brand derivation has significant typographical errors in early printings (e.g. 1947, p. 126) that are corrected in latter printings (e.g. 1954). Von Mises only states the results without derivation [28, Secs. A.7, B.8, C.1], although he does derive the derivative of the inertia dyad. Dimentberg [6] uses the principal of transference to state the moving body derivative formula with dual vectors. Yang [30] uses the dual vector representation of screws and refers to the fixed body derivative of a screw as a "pseudodual vector" since it violates the shifting law. In [31] he formulates the dynamic equations of a rigid body with the momentum screw differentiated in the moving body and shows that the formulation holds at all points.

The presentation here provides a novel and somewhat paradoxical approach using a continuum mechanics viewpoint for rigid body mechanics. This requires representing a screw quantity by a vector field [15]. It is the goal of this paper to provide the simplest explanation and clearest notation to unambiguously characterize the properties of screw differentiation with respect to time. To this end only three fundamental formulations are required: (i) the relationship between the derivatives of vectors as observed from fixed and moving bodies that is fundamental in rigid body mechanics, (ii) the relationship between the material and local derivatives of vectors that is fundamental in continuum mechanics, and (iii) the shifting law that identifies how the representation of a screw changes from point to point.

Four distinct cases of differentiation are considered that occur according to what quantities are assumed to be constant. It is shown that the material derivative of a screw with respect to a fixed body is generally not a screw quantity. This is especially important since it is the most common type of differentiation and leads to special results for Euler's Laws and the stiffness of an elastic system when expressed at the center-of-mass. However it is shown that the forms of the equations are not invariant when expressed at an arbitrary point, i.e. the shifting property is violated. Alternative screw formulations preserve invariance.

In the following development it is assumed that there are only two rigid bodies, arbitrarily referred to as the fixed and moving bodies. The formulation can be easily extended to more bodies, and for illustration is done so in an example at the end. There are also two arbitrarily selected points on the moving body, $a$ and $c$. In the case of inertial systems, it is assumed that the fixed body is replaced by an inertial body and that point $c$ is the moving body center-of-mass. All vectors are assumed to be Cartesian and the underlying space is Euclidean. The remainder of the paper is in three parts: first, the differentiation of vectors is reviewed; second, vector differentiation is applied to screw quantities, and; third, applications are discussed. 


\section{Differentiation of Cartesian vectors}

A Cartesian vector has direction and magnitude. Under rotational transformations the direction changes and the magnitude remains invariant. Fixed body, moving body, material, and local derivatives are discussed.

\subsection{Vectors}

The time derivative of vector $g(t)$ depends on how the rate of change is observed, particularly if the observation is with respect to a fixed or moving body. A simple way to express this is with vector coordinates either in frame $(i, j, k)$ rigidly attached to the fixed body or frame $\left(i^{\prime}, j^{\prime}, k^{\prime}\right)$ rigidly attached to the moving body. Differentiation with respect to an observer on the fixed body, $\frac{\mathrm{d} g(t)}{\mathrm{d} t}$, means that $(i, j, k)$ are held constant. Differentiation with respect to an observer on a moving body, $\frac{\mathrm{d}^{\prime} g(t)}{\mathrm{d} t}$, means that $\left(i^{\prime}, j^{\prime}, k^{\prime}\right)$ are held constant. A scalar however has the same derivative with respect to the fixed and moving bodies.

The time derivatives of $g(t)$ with respect to fixed and moving bodies are related by the wellknown derivative law,

$$
\frac{\mathrm{d} g(t)}{\mathrm{d} t}=\frac{\mathrm{d}^{\prime} g(t)}{\mathrm{d} t}+\omega(t) \times g(t)
$$

where $\omega$ is the angular velocity of the moving body with respect to the fixed body.

\subsection{Vector fields}

A vector field associates a vector with each point of a body, such as $g_{c}(t)$ with point $c$ of the moving body. Examples are the linear velocity field of points on a body or the bending moment field of points on a loaded cantilever beam. The time derivatives with respect to the fixed and moving bodies follow pointwise from those of vectors,

$$
\frac{\mathrm{d} g_{c}(t)}{\mathrm{d} t}=\frac{\mathrm{d}^{\prime} g_{c}(t)}{\mathrm{d} t}+\omega(t) \times g_{c}(t)
$$

It is valuable to express a vector field both as a function of time and as a function of time and a position vector $r_{c}$,

$$
g_{c}(t)=g_{c}\left(r_{c}(t), t\right)
$$

There is a subtle distinction between the two forms of $g_{c}$ that is well-known in the area of continuum mechanics but is presented here in a simplified approach and notation for brevity. For a more rigorous approach the reader is directed to basic references in continuum mechanics such as [16].

The first form, $g_{c}(t)$, is called the material or Lagrangian description where point $c$ is explicitly held constant with respect to the moving body. This description is most commonly used in rigid body mechanics where it is desired to track individual particles. For example, if $c$ represents the center-of-mass of a rigid body it is possible to measure its velocity by a tracking device. During a 
small time increment the velocity of a single particle $c$ is measured at two distinct locations, and in the limit their difference is used to determine the so-called material acceleration.

The second form, $g_{c}\left(r_{c}(t), t\right)$, is called the spatial or Eulerian description where $r_{c}(t)$ tracks the place in the fixed body currently occupied by particle $c$ of the moving body ${ }^{1}$ (see [16]). This description is most commonly used in fluid mechanics where it is difficult to track individual particles. For example, a fluid measuring device, such as for velocities, is often attached to a fixed location. During a small time increment the velocities of two different particles are measured at a single location, and in the limit their difference is used to determine the so-called local acceleration.

Differentiating $g_{c}$ with respect to the fixed body and omitting the explicit arguments gives,

$$
\frac{\mathrm{d} g_{c}}{\mathrm{~d} t}=\frac{\partial g_{c}}{\partial t}+\frac{\partial g_{c}}{\partial r_{c}} v_{c}
$$

where $v_{c}=\frac{\mathrm{d} r_{c}}{\mathrm{~d} t}$ is the velocity of point $c$ with respect to the fixed body. This holds for both rigid and deformable bodies. Assuming rigid bodies and differentiating with respect to the moving body gives,

$$
\frac{\mathrm{d}^{\prime} g_{c}}{\mathrm{~d} t}=\frac{\partial^{\prime} g_{c}}{\partial t}
$$

since $\frac{\partial^{\prime} g_{c}}{\partial r_{c}} v_{c}^{\prime}$ vanishes because the velocity of $c$ with respect to the moving body is zero, $v_{c}^{\prime}=0$.

The operators $\frac{\mathrm{d}}{\mathrm{d} t}$ and $\frac{\mathrm{d}^{\prime}}{\mathrm{d} t}$ are known as the material or total derivatives with respect to the fixed and moving bodies respectively. The operators $\frac{\partial}{\partial t}$ and $\frac{\frac{\partial}{\prime}^{\prime} t}{\partial t}$ are known as the local or spatial derivatives with respect to the fixed and moving bodies respectively. It is emphasized that while the material derivative exists for both an isolated particle and a continuum, the spatial derivative exists only for a continuum.

Example 1. Fig. 1 shows two particles on the edge of a rigid disk rotating about a fixed axis with an angular acceleration. In Fig. 1(a) $r_{c}(t)$ points to the place in the fixed body (not shown) currently occupied by point $c$ with velocity $v_{c}\left(r_{c}(t), t\right)$. In Fig. 1(b) the disk has turned and a new particle with velocity $v_{c}\left(r_{c}(t), t+\mathrm{d} t\right)$ now occupies $r_{c}(t)$, the position previously occupied by $c$. In the limit the change in velocity is $\frac{\partial v_{c}}{\partial t} \mathrm{~d} t$ where $\frac{\partial v_{c}}{\partial t}$ is the local acceleration. In Fig. 1(c) $r_{c}(t+\mathrm{d} t)$ points to the place in the fixed body currently occupied by the new position of particle $c$. In the limit the change in velocity is $\frac{\mathrm{d} v_{c}}{\mathrm{~d} t} \mathrm{~d} t$ where $\frac{\mathrm{d} v_{c}}{\mathrm{~d} t}$ is the material acceleration. Note that in this example: the local acceleration is also the tangential component of the material acceleration; in the special instance of no angular acceleration, the local acceleration vanishes, $\frac{\partial v_{c}}{\partial t}=0$, and the material acceleration, $\frac{\mathrm{d} v_{c}}{\mathrm{~d} t}$, becomes the centripetal acceleration to the center of the disk.

\section{Screw quantities}

A screw quantity can be conveniently defined in terms of Cartesian vectors and the shifting property,

\footnotetext{
${ }^{1}$ This seemingly awkward device is required to justify the existence of partial derivatives in $t$ and $r_{c}$.
} 

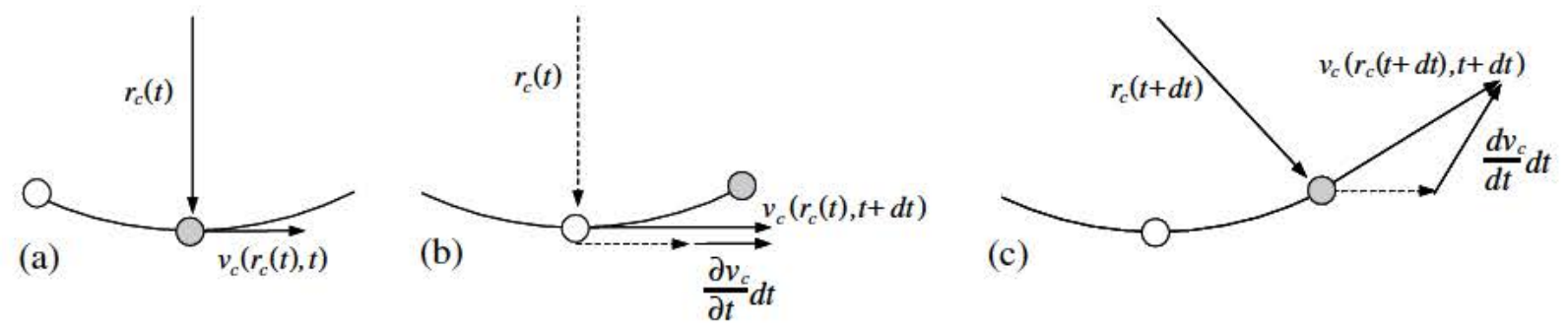

Fig. 1. Two particles on the edge of a rotating disk (a) illustrate the local acceleration (b) and the material acceleration (c).

Definition 2. The vector pair $\left(g, g_{c}\right)$ represents a screw quantity if and only if it satisfies the shifting property

$$
g_{a}=r_{a c} \times g+g_{c}
$$

for every pair of points $a$ and $c$ of the moving body where $r_{a c}$ is the vector from $a$ to $c$, and where $g, g_{c}, g_{a}, r_{a c}$ are Cartesian vectors.

As a point of reference, the shifting law is often expressed as the linear relation,

$$
\left[\begin{array}{l}
g \\
g_{a}
\end{array}\right]=\left[\begin{array}{cc}
1 & 0 \\
r_{a c} \times & 1
\end{array}\right]\left[\begin{array}{c}
g \\
g_{c}
\end{array}\right]
$$

where 1 is a $3 \times 3$ identity matrix and $r_{a c} \times$ is the $3 \times 3$ skew-symmetric cross product operator. The screw quantity is written in the form known as Plücker ray coordinates. (When the order of $g$ and $g_{c}$ are reversed they are known as Plücker axis coordinates.) Satisfying the shifting law simply means that the vector field undergoes the same type of rigid body transformation as velocities or loads as shown in the following examples.

Example 3. Two basic screw quantities are $\left(\omega, v_{c}\right)$ - the velocity twist of rigid body, and $\left(f, m_{c}\right)$ the load wrench on a rigid body. At point $a$, the equivalent properties are specified respectively as $\left(\omega, v_{a}\right)=\left(\omega, r_{a c} \times \omega+v_{c}\right)$ and $\left(f, m_{a}\right)=\left(f, r_{a c} \times f+m_{c}\right)$.

Example 4. The momentum of a rigid body $\left(p, h_{c}\right)$ is a screw quantity. The linear momentum is given by $p=m v_{c}$, where $m$ is the mass and $c$ is the center-of-mass. The angular momentum about $c$ is $h_{c}=I_{c} \omega$, where $I_{c}$ is the inertia matrix about $c$. The equivalent properties at point $a$ on the body are $\left(p, h_{a}\right)=\left(p, r_{a c} \times p+h_{c}\right)=\left(m v_{c}, r_{a c} \times m v_{c}+I_{c} \omega\right) .^{2}$

\footnotetext{
${ }^{2}$ Note that linear momentum is $p \equiv \int_{v} \mathrm{~d} m \quad m v_{c}$, where $v$ is the inertial velocity of particle $\mathrm{d} m$, although often it is expressed equivalently as $m r_{c a} \times \omega+m v_{a}$. Angular momentum is $h_{a} \equiv \int r \times v \mathrm{~d} m$, where $r$ points from $a$ to each particle. Unfortunately, a few authors use "relative" angular momentum, $\int r \times\left(\begin{array}{ll}v & v_{a}\end{array}\right) \mathrm{d} m$ which, together with linear momentum, does not satisfy the shifting property so it is not a screw quantity. A special result occurs at the center of mass $c$ where

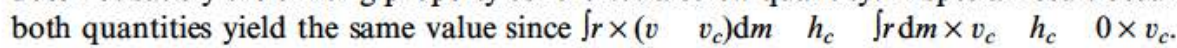




\section{Differentiation of screws}

Four cases of differentiating a screw are examined by using material or local derivatives with respect to moving or fixed bodies. One case does not yield a screw quantity and two cases yield the same screw quantity.

\subsection{Material derivative-moving body}

Differentiating the shifting law with respect to the moving body gives,

$$
\frac{\mathrm{d}^{\prime} g_{a}}{\mathrm{~d} t}=r_{a c} \times \frac{\mathrm{d}^{\prime} g}{\mathrm{~d} t}+\frac{\mathrm{d}^{\prime} g_{c}}{\mathrm{~d} t}
$$

where $r_{a c}$ is constant with respect to the moving body. However this is just the shifting law for $\frac{\mathrm{d}^{\prime}}{\mathrm{d} t}\left(g, g_{c}\right)$ so the following has been shown,

Proposition 5. For a screw quantity $\left(g, g_{c}\right)$ where $c$ is a point of the moving body, the material derivative with respect to the moving body $\frac{\mathrm{d}^{\prime}}{\mathrm{d} t}\left(g, g_{c}\right)$ is a screw quantity.

It is also useful to express this quantity in terms of fixed body material derivatives,

$$
\left(\frac{\mathrm{d}^{\prime} g}{\mathrm{~d} t}, \frac{\mathrm{d}^{\prime} g_{c}}{\mathrm{~d} t}\right)=\left(\begin{array}{ll}
\frac{\mathrm{d} g}{\mathrm{~d} t} \quad \omega \times g, \frac{\mathrm{d} g_{c}}{\mathrm{~d} t} \quad \omega \times g_{c}
\end{array}\right)
$$

Example 6. For a twist $\left(g, g_{c}\right)=\left(\omega, v_{c}\right)$,

$$
\left(\frac{\mathrm{d}^{\prime} \omega}{\mathrm{d} t}, \frac{\mathrm{d}^{\prime} v_{c}}{\mathrm{~d} t}\right)=\left(\begin{array}{ll}
\frac{\mathrm{d} \omega}{\mathrm{d} t} \quad \omega \times \omega, \frac{\mathrm{d} v}{\mathrm{~d} t} \quad \omega \times v_{c}
\end{array}\right)=\left(\alpha, a_{c} \quad \omega \times v_{c}\right)
$$

where $\alpha$ is the angular acceleration and $a_{c}$ is the linear acceleration of point $c$. This is an important special case where one of the terms, $\omega \times \omega$, cancels out.

Example 7. For a wrench $\left(g, g_{c}\right)=\left(f, m_{c}\right)$,

$$
\left(\frac{\mathrm{d}^{\prime} f}{\mathrm{~d} t}, \frac{\mathrm{d}^{\prime} m_{c}}{\mathrm{~d} t}\right)=\left(\begin{array}{ll}
\frac{\mathrm{d} f}{\mathrm{~d} t} \quad \omega \times f, \frac{\mathrm{d} m_{c}}{\mathrm{~d} t} \quad \omega \times m_{c}
\end{array}\right)
$$

Unlike acceleration, this is indicative of the generic case since $\omega \times f$ does not generally vanish.

\subsection{Local derivative - moving body}

Since $g(t)$ is not a function of $r_{c}$ then $\frac{\mathrm{d}^{\prime} g}{\mathrm{~d} t}=\frac{\partial^{\prime} g}{\partial t}$. Also in Section 2.2 it was shown that $\frac{\mathrm{d}^{\prime} g_{c}}{\mathrm{~d} t}=\frac{\partial^{\prime} g_{c}}{\partial t}$ since $v_{c}^{\prime}=0$. Together with Proposition 5 this gives,

Proposition 8. For a screw quantity $\left(g, g_{c}\right)$ where $c$ is a point of the moving body, the local derivative with respect to the moving body $\frac{\partial^{\prime}}{\partial t}\left(g, g_{c}\right)$ is a screw quantity, and further $\frac{\partial^{\prime}}{\partial t}\left(g, g_{c}\right)=\frac{\mathrm{d}^{\prime}}{\mathrm{d} t}\left(g, g_{c}\right)$.

Thus the local and material derivatives with respect to the moving body are equal. 


\subsection{Material derivative- fixed body}

This is the most important case. If $\left(g, g_{c}\right)$ is a screw quantity then in general $\frac{\mathrm{d}}{\mathrm{d} t}\left(g, g_{c}\right)$ is not a screw quantity since it does not satisfy the shifting property. This is easy to show by differentiating the shifting law,

$$
\frac{\mathrm{d} g_{a}}{\mathrm{~d} t}=\frac{\mathrm{d} r_{a c}}{\mathrm{~d} t} \times g+r_{a c} \times \frac{\mathrm{d} g}{\mathrm{~d} t}+\frac{\mathrm{d} g_{c}}{\mathrm{~d} t}
$$

For $\frac{\mathrm{d}}{\mathrm{d} t}\left(g, g_{c}\right)$ to satisfy the shifting law it is necessary to satisfy the condition $\frac{\mathrm{d} r a c}{\mathrm{~d} t} \times g=0$ for every pair of points $a$ and $c$ on the moving body which implies that $g=0$ or that the body is translating, $\frac{\mathrm{d} r_{a c}}{\mathrm{~d} t}=0$.

Proposition 9. For a screw quantity $\left(g, g_{c}\right)$ where $c$ is a point of the moving body, the material derivative with respect to the fixed body $\frac{\mathrm{d}}{\mathrm{d} t}\left(g, g_{c}\right)$ is generally not a screw quantity, except for the special cases where $g=0$ or the body is translating.

Example 10. For a twist $\left(g, g_{a}\right)=\left(\omega, v_{a}\right)$,

$$
\frac{\mathrm{d}}{\mathrm{d} t}\left(\omega, v_{a}\right)=\left(\frac{\mathrm{d} \omega}{\mathrm{d} t}, \frac{\mathrm{d}}{\mathrm{d} t}\left(r_{a c} \times \omega+v_{c}\right)\right)=\left(\alpha, a_{c}+\alpha \times r_{c a}+\omega \times\left(\omega \times r_{c a}\right)\right)
$$

This is a well-known relation in kinematics. For $\frac{\mathrm{d}}{\mathrm{d} t}\left(\omega, v_{a}\right)$ to be a screw quantity the body must be instantaneously translating, $\omega=0$, so the centripetal acceleration of $a$ about $c$ vanishes, $\omega \times\left(\omega \times r_{c a}\right)=0$, for all such pairs of points. In this particular example the two special cases of Proposition 9 are the same.

It is necessary to examine the field viewpoint of the material derivative,

$$
\frac{\mathrm{d}}{\mathrm{d} t}\left(g, g_{c}\right)=\left(\frac{\partial g}{\partial t}+\frac{\partial g}{\partial r_{c}} v_{c}, \frac{\partial g_{c}}{\partial t}+\frac{\partial g_{c}}{\partial r_{c}} v_{c}\right)
$$

To evaluate $\frac{\partial g}{\partial r_{c}}$ note that $g=g(t)$ does not depend on $r_{c}$ so $\frac{\partial g}{\partial r_{c}}=0$. To evaluate $\frac{\partial g_{c}}{\partial r_{c}}$ the shifting law is expressed as $g_{a}=g \times\left(\begin{array}{ll}r_{a} & r_{c}\end{array}\right)+g_{c}$ and the partial is taken with respect to $r_{c}$ to give

$$
\frac{\partial g_{c}}{\partial r_{c}}=g \times
$$

where $g, g_{a}, r_{a}$ are not functions of $r_{c}$. These results yield the material derivative of a screw as

$$
\frac{\mathrm{d}}{\mathrm{d} t}\left(g, g_{c}\right)=\left(\frac{\partial g}{\partial t}, \frac{\partial g_{c}}{\partial t}+g \times v_{c}\right)
$$

which is generally not a screw quantity unless $g=0$ or the body is translating. This expression is used frequently throughout the sequel. 


\subsection{Local derivative- fixed body}

Again using the material derivative of the shifting law but now substituting in the partials gives,

$$
\begin{aligned}
& \frac{\mathrm{d} g_{a}}{\mathrm{~d} t}=\frac{\mathrm{d} r_{a c}}{\mathrm{~d} t} \times g+r_{a c} \times \frac{\mathrm{d} g}{\mathrm{~d} t}+\frac{\mathrm{d} g_{c}}{\mathrm{~d} t} \\
& \left(\frac{\partial g_{a}}{\partial t}+g \times v_{a}\right)=\left(\begin{array}{ll}
v_{c} & \left.v_{a}\right) \times g+r_{a c} \times \frac{\partial g}{\partial t}+\left(\frac{\partial g_{c}}{\partial t}+g \times v_{c}\right.
\end{array}\right) \\
& \frac{\partial g_{a}}{\partial t}=r_{a c} \times \frac{\partial g}{\partial t}+\frac{\partial g_{c}}{\partial t}
\end{aligned}
$$

However the last expression is just the shifting law for $\frac{\partial}{\partial t}\left(g, g_{c}\right)$ so the following has been shown,

Proposition 11. For a screw quantity $\left(g, g_{c}\right)$ where $c$ is a point of the moving body, the local derivative with respect to the fixed body $\frac{\partial}{\partial t}\left(g, g_{c}\right)$ is also a screw quantity.

It is also useful to express this quantity in terms of fixed body material derivatives,

$$
\left(\frac{\partial g}{\partial t}, \frac{\partial g_{c}}{\partial t}\right)=\left(\frac{\mathrm{d} g}{\mathrm{~d} t}, \frac{\mathrm{d} g_{c}}{\mathrm{~d} t} \quad g \times v_{c}\right)
$$

Example 12. For a twist $\left(g, g_{c}\right)=\left(\omega, v_{c}\right)$,

$$
\left(\frac{\partial \omega}{\partial t}, \frac{\partial v_{c}}{\partial t}\right)=\left(\frac{\mathrm{d} \omega}{\mathrm{d} t}, \frac{\mathrm{d} v}{\mathrm{~d} t} \quad \omega \times v_{c}\right)=\left(\alpha, a_{c} \quad \omega \times v_{c}\right)
$$

This is the same result as in Example 6, where $\omega \times \omega$ cancelled out in the calculation of $\frac{\mathrm{d}^{\prime} \omega}{\mathrm{d} t}$ and thus $\frac{\partial}{\partial t}\left(\omega, v_{c}\right)=\frac{\mathrm{d}^{\prime}}{\mathrm{d} t}\left(\omega, v_{c}\right)$. This is a special situation that only occurs for acceleration. The term $a_{c}^{l} \equiv a_{c} \quad \omega \times v_{c}$ is the local acceleration.

Example 13. For a wrench $\left(g, g_{c}\right)=\left(f, m_{c}\right)$,

$$
\left(\frac{\partial f}{\partial t}, \frac{\partial m_{c}}{\partial t}\right)=\left(\frac{\mathrm{d} f}{\mathrm{~d} t}, \frac{\mathrm{d} m_{c}}{\mathrm{~d} t} \quad f \times v_{c}\right)
$$

In contrast to the acceleration case, this result is distinct from Example 7 so $\frac{\partial}{\partial t}\left(f, m_{c}\right) \neq \frac{\mathrm{d}^{\prime}}{\mathrm{d} t}\left(f, m_{c}\right)$ and is representative of the general situation.

Example 14. For momentum $\left(g, g_{a}\right)=\left(p, h_{a}\right)$,

$$
\left(\frac{\partial p}{\partial t}, \frac{\partial h_{a}}{\partial t}\right)=\left(\frac{\mathrm{d} p}{\mathrm{~d} t}, \frac{\mathrm{d} h_{a}}{\mathrm{~d} t} \quad p \times v_{a}\right)
$$

where $a$ is an arbitrary point of the body. This result is similar to the general wrench case. However, in the particular for the center-of-mass, 
Table 1

Derivatives of screw quantities expressed using fixed body material derivatives

\begin{tabular}{|c|c|c|}
\hline & Material derivative & Local derivative \\
\hline Fixed body & $\frac{\mathrm{d}}{\mathrm{d} t}\left(g, g_{c}\right)=\left(\frac{\mathrm{d} g}{\mathrm{~d} t}, \frac{\mathrm{d} g_{c}}{\mathrm{~d} t}\right)$ (not a screw) & $\frac{\partial}{\partial t}\left(g, g_{c}\right)=\left(\frac{\mathrm{d} g}{\mathrm{~d} t}, \frac{\mathrm{d} g_{c}}{\mathrm{~d} t} \quad g \times v_{c}\right)$ \\
\hline Moving body & 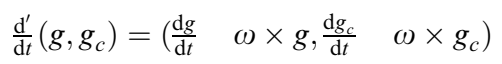 & $\frac{\partial^{\prime}}{\partial t}\left(g, g_{c}\right)=\left(\frac{\mathrm{d} g}{\mathrm{~d} t}\right.$ \\
\hline
\end{tabular}

$$
\left(\frac{\partial p}{\partial t}, \frac{\partial h_{c}}{\partial t}\right)=\left(\frac{\mathrm{d} p}{\mathrm{~d} t}, \frac{\mathrm{d} h_{c}}{\mathrm{~d} t}\right)
$$

since $p \times v_{c}=m v_{c} \times v_{c}=0$ and so $\frac{\partial}{\partial t}\left(p, h_{c}\right)=\frac{\mathrm{d}}{\mathrm{d} t}\left(p, h_{c}\right)$. This is a another special instance of cancellation and only occurs when the angular momentum is expressed at the center-of-mass. However this instance is a very important case in dynamics.

\subsection{Summary of cases}

In the previous sections the alternative expressions for the various derivatives were developed using material derivatives in the fixed frame. These are particularly useful for calculation since they are the most basic derivatives in rigid body mechanics. For ease of comparison and reference the expressions are collected in Table 1.

\subsection{Local derivatives connecting fixed and moving bodies}

The previous results on local derivatives can be combined to give the connection between the local derivatives of screws with respect to moving and fixed bodies,

$$
\begin{aligned}
\left(\frac{\partial g}{\partial t}, \frac{\partial g_{c}}{\partial t}\right) & =\left(\frac{\mathrm{d} g}{\mathrm{~d} t}, \frac{\mathrm{d} g_{c}}{\mathrm{~d} t} \quad g \times v_{c}\right)=\left(\frac{\mathrm{d}^{\prime} g}{\mathrm{~d} t}+\omega \times g, \frac{\mathrm{d}^{\prime} g_{c}}{\mathrm{~d} t}+\omega \times g_{c} \quad g \times v_{c}\right) \\
& =\left(\frac{\partial^{\prime} g}{\partial t}+\omega \times g, \frac{\partial^{\prime} g_{c}}{\partial t}+\omega \times g_{c}+v_{c} \times g\right) \\
& =\left(\frac{\partial^{\prime} g}{\partial t}, \frac{\partial^{\prime} g_{c}}{\partial t}\right)+\left(\omega \times g, \omega \times g_{c}+v_{c} \times g\right)=\left(\frac{\partial^{\prime} g}{\partial t}, \frac{\partial^{\prime} g_{c}}{\partial t}\right)+\left(\omega, v_{c}\right) \times\left(g, g_{c}\right)
\end{aligned}
$$

where in the last term the screw cross product is defined as

$$
\left(e, e_{c}\right) \times\left(g, g_{c}\right)=\left(e \times g, e \times g_{c}+e_{c} \times g\right)
$$

The screw cross product yields a screw since it is direct to show that it satisfies the shifting law,

$$
\left(e \times g_{a}+e_{a} \times g\right)=r_{a c} \times(e \times g)+\left(e \times g_{c}+e_{c} \times g\right)
$$

As a point of reference, the screw cross product is equivalent to the Lie bracket of screw quantities. The results are summarized as,

Proposition 15. For a screw quantity $\left(g, g_{c}\right)$ where $c$ is a point of the moving body, the fixed and moving local derivatives are related by 


$$
\frac{\partial}{\partial t}\left(g, g_{c}\right)=\frac{\partial^{\prime}}{\partial t}\left(g, g_{c}\right)+\left(\omega, v_{c}\right) \times\left(g, g_{c}\right)
$$

This relation is rather remarkable in form because it generalizes the vector relation $\frac{\mathrm{d}}{\mathrm{d} t} g=\frac{\mathrm{d}^{\prime}}{\mathrm{d} t} g+\omega \times g$.

\section{Applications}

Two fundamental applications are considered, the dynamics of a rigid body and the stiffness of an elastically suspended rigid body. Special results occur when they are formulated at the centerof-mass.

\subsection{Euler's Laws}

Euler's 1st and 2nd Laws provide the six dynamic equations for an unconstrained rigid body in space. ${ }^{3}$ These are combined as $\left(f, m_{c}\right)=\frac{\mathrm{d}}{\mathrm{d} t}\left(p, h_{c}\right)$ where $c$ is the center-of-mass and $p, h_{c}$ are the linear momentum and angular momentum as in Example 4. However there appears to be an inconsistency because the wrench $\left(f, m_{c}\right)$ is identified as a screw quantity in Example 3 while Proposition 9 shows that the material derivative $\frac{\mathrm{d}}{\mathrm{d} t}\left(p, h_{c}\right)$ is generally not a screw quantity because the shifting law is not satisfied.

The resolution is in Example 14 where the fortuitous cancellation $p \times v_{c}=0$ makes the nonscrew quantity $\frac{\mathrm{d}}{\mathrm{d} t}\left(p, h_{c}\right)$ equivalent to the screw quantity $\frac{\partial}{\partial t}\left(p, h_{c}\right)$, but only at the center-of-mass $c$. At an arbitrary point $a$ of the body a similar cancellation does not occur for the material derivative, $\left(f, m_{a}\right) \neq \frac{\mathrm{d}}{\mathrm{d} t}\left(p, h_{a}\right)$, however it is direct to show that Euler's Laws retain the correct form for the local derivative, $\left(f, m_{a}\right)=\frac{\partial}{\partial t}\left(p, h_{a}\right)$. Thus the local derivative yields the most general form of Euler's Laws. The properties are summarized in Table 2.

From Proposition 15 Euler's Laws can be expressed using a derivative with respect to the moving body at point $a$,

$$
\left(f, m_{a}\right)=\frac{\partial^{\prime}}{\partial t}\left(p, h_{a}\right)+\left(\omega, v_{a}\right) \times\left(p, h_{a}\right)
$$

which generalizes the familiar form of Euler's 2nd Law expressed at the center-of-mass, $m_{c}=\frac{\mathrm{d}^{\prime}}{\mathrm{d} t} h_{c}+\omega \times h_{c}=I_{c} \alpha+\omega \times I_{c} \omega$.

It is also useful to represent Euler's Laws in a matrix form, and for simplicity is first done at the center-of-mass,

$$
\left[\begin{array}{c}
f \\
m_{c}
\end{array}\right]=\left[\begin{array}{cc}
m 1 & 0 \\
0 & I_{c}
\end{array}\right]\left[\begin{array}{c}
a_{c}^{l} \\
\alpha
\end{array}\right]+\left[\begin{array}{c}
v_{c} \\
\omega
\end{array}\right] \times\left[\begin{array}{cc}
m 1 & 0 \\
0 & I_{c}
\end{array}\right]\left[\begin{array}{c}
v_{c} \\
\omega
\end{array}\right]
$$

\footnotetext{
${ }^{3}$ Newton's 2nd Law, $f=\frac{\mathrm{d}}{\mathrm{d} t} m v$, applies to particles and point masses whereas Euler's 1st Law, $f=\frac{\mathrm{d}}{\mathrm{d} t} m v_{c}$, applies to bodies.
} 
Table 2

Properties of Euler's Laws

\begin{tabular}{lll}
\hline & Material derivative & Local derivative \\
\hline Mass center & $\left(f, m_{c}\right)=\frac{\mathrm{d}}{\mathrm{d} t}\left(p, h_{c}\right)$ & $\left(f, m_{c}\right)=\frac{\partial}{\partial t}\left(p, h_{c}\right)$ \\
Arbitrary point & $\left(f, m_{a}\right) \neq \frac{\mathrm{d}}{\mathrm{d} t}\left(p, h_{a}\right)$ & $\left(f, m_{a}\right)=\frac{\partial}{\partial t}\left(p, h_{a}\right)$
\end{tabular}

where the applied wrench is in Plücker ray coordinates, the velocity and acceleration twists are in Plücker axis coordinates, and the screw cross product must be defined in a manner consistent with this representation. The term $a_{c}^{l}$ is the linear local acceleration as defined in Example 12 and $I_{c}$ is constant with respect to the moving body. A more condensed expression, using $\hat{f}, \hat{a}^{l}$, and $\hat{v}$ to denote screw quantities, is $\hat{f}_{c}=M_{c} \hat{a}_{c}^{l}+\hat{v}_{c} \times M_{c} \hat{v}_{c}$. At an arbitrary point $a$ of the body the dynamic equations have the same form $\hat{f}_{a}=M_{a} \hat{a}_{a}^{l}+\hat{v}_{a} \times M_{a} \hat{v}_{a}$, where, if $T_{a c}$ represents the matrix operator of the shifting law for Plücker ray coordinates as in Section 3 then, $\hat{f}_{a}=T_{a c} \hat{f}_{c}$, $\hat{a}_{a}^{l}=T_{a c}^{\mathrm{T}} \hat{a}_{c}^{l}, \hat{v}_{a}=T_{a c}^{\mathrm{T}} \hat{v}_{c}$, and the congruence transformation $M_{a}=T_{a c} M_{c} T_{a c}^{\mathrm{T}}$ is the generalization of the parallel axis theorem for screw inertias.

\subsection{Stiffness}

An elastically suspended rigid body can be modeled using screw quantities [9,5]. Consider $n$ line springs, each attached to the moving body at point $c_{i}$ with the other end attached to the fixed body (see Fig. 2). For each spring the stiffness is $k_{i}$, the length is $l_{i}$, the undeformed length is $l_{0 i}$, and the direction is $e_{i}$. Point $a$ on the rigid body is the location of an external wrench that loads the system in static equilibrium.

In this type of problem, it is commonplace to introduce differentials $\mathrm{d}(\cdot)$ rather than time derivatives $\frac{\mathrm{d}(\cdot)}{\mathrm{d} t}$. A small displacement of the moving body changes the springs by $\mathrm{d}\left(l_{i} e_{i}\right)=\mathrm{d} r_{c_{i}}$ and together with $\mathrm{d}\left(l_{i} e_{i}\right)=l_{i} \mathrm{~d} e_{i}+e_{i} \mathrm{~d} l_{i}$ yields the kinematic relations

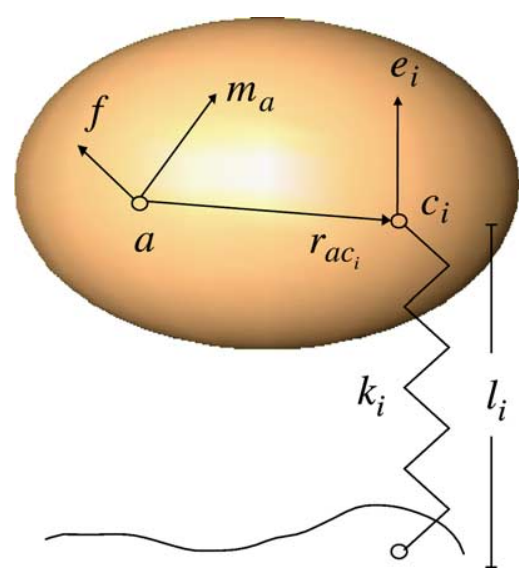

Fig. 2. An elastically suspended rigid body (one spring shown). 


$$
\begin{aligned}
e_{i} \mathrm{~d} l_{i} & =e_{i} e_{i}^{\mathrm{T}} \mathrm{d} r_{c_{i}} \\
l_{i} \mathrm{~d} e_{i} & =e_{i} \times e_{i} \times \mathrm{d} r_{c_{i}}
\end{aligned}
$$

The displacement shifting law becomes $\mathrm{d} r_{a}=r_{a c_{i}} \times \mathrm{d} \theta+\mathrm{d} r_{c_{i}}$ where $\mathrm{d} \theta$ is a small rotation of the body (but is not a true differential). The force applied to each spring is $f_{i}=k_{i}\left(l_{i} \quad l_{0 i}\right)$ so the net wrench applied to the moving body at $a$ is $\left(f, m_{a}\right)=\sum\left(f_{i}, r_{a c_{i}} \times f_{i}\right)$. Taking the material differential of the wrench with respect to the fixed body, using the kinematic relations and shifting law to simplify, and arranging in a matrix form yields,

$$
\left[\begin{array}{c}
\mathrm{d} f \\
\mathrm{~d} m_{a}
\end{array}\right]=K\left[\begin{array}{c}
\mathrm{d} r_{a} \\
\mathrm{~d} \theta
\end{array}\right]
$$

where $K$ is the $6 \times 6$ material stiffness matrix written as the sum of three parts,

$$
\begin{aligned}
& K=K_{1}+K_{2}+K_{3} \\
& K_{1}=\sum_{i} k_{i}\left[\begin{array}{c}
e_{i} \\
r_{a c_{i}} \times e_{i}
\end{array}\right]\left[\begin{array}{c}
e_{i} \\
r_{a c_{i}} \times e_{i}
\end{array}\right]^{\mathrm{T}} \\
& K_{2}=\sum_{i} k_{i}\left(1 \quad \rho_{i}\right)\left[\begin{array}{c}
e_{i} \times \\
r_{a c_{i}} \times e_{i} \times
\end{array}\right]\left[\begin{array}{c}
e_{i} \times \\
r_{a c_{i}} \times e_{i} \times
\end{array}\right]^{\mathrm{T}} \\
& K_{3}=\sum_{i}\left[\begin{array}{cc}
0 & 0 \\
0 & f_{i} \times r_{a c_{i}} \times
\end{array}\right]
\end{aligned}
$$

and where $\rho_{i} \equiv l_{0 i} / l_{i}$. Terms $K_{1}$ and $K_{2}$ are symmetric while $K_{3}$ is not. When all the springs are unloaded, $\rho_{i}=1$ and $f_{i}=0$ so $K_{2}$ and $K_{3}$ vanish. The stiffness can also be expressed as the sum of symmetric and skew-symmetric parts, $K=K_{\text {sym }}+K_{\text {skew }}$. Using the Jacobi identity, $(a \times b) \times=$ $(a \times b \times) \quad(b \times a \times)$, the skew part reduces to the simple form,

$$
K_{\text {skew }}=\frac{1}{2}\left[\begin{array}{cc}
0 & 0 \\
0 & m_{a} \times
\end{array}\right]
$$

Therefore the applied moment accounts for the skew-symmetry; when the system is unloaded, the stiffness is symmetric.

Though the material stiffness relation is correct, $\mathrm{d}\left(f, m_{a}\right)$ is not a screw quantity. As a consequence, when changing to another point the stiffness matrix $K$ does not transform by the congruence transformation given in Section 5.1 and hence the form of $K$ is not invariant. For example, consider the important special case where the external load is due to the gravity load of the suspended body. At the center-of-mass $c$ the moment vanishes, $m_{c}=0$, and the material stiffness is symmetric. If another point $a$ on the body is selected, then generally $m_{a} \neq 0$ and the material stiffness has a skew-symmetric component. This highlights a deficiency of material stiffness-the form of the matrix representation is dependent on the selected point, i.e. it is not invariant.

However, a stiffness relation based on a local differential operation does yield an invariant expression amongst screw quantities. Introducing a differential operator defined by the local time derivative, $\partial(\cdot) \equiv \frac{\partial(\cdot)}{\partial t} \mathrm{~d} t$, then $\left(\mathrm{d} f, \mathrm{~d} m_{a}\right)=\left(\partial f, \partial m_{a}+f \times \mathrm{d} r_{a}\right)$. Substituting this into the material stiffness expression yields the corresponding local stiffness expression, 


$$
\left[\begin{array}{c}
\partial f \\
\partial m_{a}
\end{array}\right]=K^{l}\left[\begin{array}{c}
\mathrm{d} r_{a} \\
\mathrm{~d} \theta
\end{array}\right]
$$

where $K^{l}=K_{1}+K_{2}+K_{3}^{l}$ and

$$
K_{3}^{l}=\sum_{i}\left[\begin{array}{cc}
0 & 0 \\
f_{i} \times & f_{i} \times r_{a c_{i}} \times
\end{array}\right]
$$

Expressing the local stiffness in symmetric and skew-symmetric parts, $K^{l}=K_{\text {sym }}+K_{\text {skew }}^{l}$, gives [5],

$$
K_{\text {skew }}^{l}=\frac{1}{2}\left[\begin{array}{cc}
0 & f \times \\
f \times & m_{a} \times
\end{array}\right] \equiv \frac{1}{2}\left(f, m_{a}\right) \times
$$

where $\left(f, m_{a}\right) \times$ is a form of the screw cross product operator which in this case is a transformation from Plücker axis coordinates to Plücker ray coordinates. ${ }^{4}$

The result is rather remarkable because it shows that the skew-symmetry of the local stiffness matrix is only dependent on the external wrench; therefore, the stiffness matrix is symmetric if and only if the system is at an unloaded equilibrium. For the gravity load case, the local stiffness $K^{l}$ always has a skew-symmetric part no matter which point on the body is selected. Thus in contrast to material stiffness, the form of the local stiffness matrix is invariant with respect to the selected point. As a consequence the local stiffness undergoes a congruence transformation, $\left(K^{l}\right)_{a}=T_{a c}\left(K^{l}\right)_{c} T_{a c}^{\mathrm{T}}$ (subscripts added for emphasis), whereas the material derivative does not.

The moving body material and local the stiffness matrices are the same since $\mathrm{d}^{\prime}\left(f, m_{a}\right)=\partial^{\prime}\left(f, m_{a}\right)$. This stiffness is determined from Proposition 15, $\partial\left(f, m_{a}\right)=\partial^{\prime}\left(f, m_{a}\right)+\left(\mathrm{d} r_{a}, \mathrm{~d} \theta\right) \times\left(f, m_{a}\right)$, and substitution into the previous local stiffness relation with $\left(\mathrm{d} r_{a}, \mathrm{~d} \theta\right) \times\left(f, m_{a}\right)=\left(f, m_{a}\right) \times\left(\mathrm{d} r_{a}, \mathrm{~d} \theta\right)$ to give the moving body local stiffness as

$$
\left[\begin{array}{c}
\partial^{\prime} f \\
\partial^{\prime} m_{a}
\end{array}\right]=K^{\prime \prime}\left[\begin{array}{c}
\mathrm{d} r_{a} \\
\mathrm{~d} \theta
\end{array}\right]
$$

where $K^{\prime \prime}=\left(K^{1}\right)^{\mathrm{T}}=K_{\text {sym }}+\left(K_{\text {skew }}^{1}\right)^{\mathrm{T}}=K_{\text {sym }}+\frac{1}{2}\left(f, m_{a}\right) \times$. Thus the fixed and moving body stiffnesses are simply transposes of each other. This was shown in [19] for the planar case and extended to the spatial case in [5].

A short example from [10] illustrates the extension of the formulation to more than two bodies. Consider a third body that is moving with respect to the other two. The local derivatives observed with respect to the fixed and third bodies are related by Proposition 15 as $\partial\left(f, m_{a}\right)=$ $\mathrm{\partial}^{\prime \prime}\left(f, m_{a}\right)+\left(\mathrm{d} r_{a}^{\prime \prime}, \mathrm{d} \theta^{\prime \prime}\right) \times\left(f, m_{a}\right)$ where $\left(\mathrm{d} r_{a}^{\prime \prime}, \mathrm{d} \theta^{\prime \prime}\right)$ is the motion of the third body with respect to the fixed body and $\partial^{\prime \prime}(\cdot)$ is the derivative with respect to the third body. Expressing the fixed body local stiffness relation as, $\partial\left(f, m_{a}\right)=\left(K_{\text {sym }} \frac{1}{2}\left(f, m_{a}\right) \times\right)\left(\mathrm{d} r_{a}, \mathrm{~d} \theta\right)$ and eliminating $\partial\left(f, m_{a}\right)$ yields,

$$
\mathrm{\partial}^{\prime \prime}\left(f, m_{a}\right)=K_{\mathrm{sym}}\left(\mathrm{d} r_{a}, \mathrm{~d} \theta\right)+\left(f, m_{a}\right) \times\left(\left(\mathrm{d} r_{a}^{\prime \prime}, \mathrm{d} \theta^{\prime \prime}\right) \quad \frac{1}{2}\left(\mathrm{~d} r_{a}, \mathrm{~d} \theta\right)\right)
$$

\footnotetext{
${ }^{4}$ Note that the matrix form of the screw cross product operator is dependent on the specific combination of axis and ray coordinates used.
} 
If the motion of the third body is half as fast as the moving body, $\left(\mathrm{d} r_{a}^{\prime \prime}, \mathrm{d} \theta^{\prime \prime}\right)=\frac{1}{2}\left(\mathrm{~d} r_{a}, \mathrm{~d} \theta\right)$, then the resulting stiffness matrix is just the symmetric part $K_{\text {sym }}$.

\section{Concluding remarks}

In this exposition, a basic continuum mechanics formulation facilitates identification of properties for various time derivatives of a screw quantity; an unambiguous derivative notation helps make the differences evident. Local differentiation always produces a screw quantity and, with respect to rigid body formulations, could rightly be called "screw differentiation." Two applications have been identified where initially it appears that the fixed body material derivative could yield the most general formulation. However, these are shown to be dependent on using the center-ofmass and the same results do not apply when formulated at an arbitrary point.

\section{References}

[1] R.S. Ball, A Treatise on the Theory of Screws, Cambridge University Press, 1900 (reprinted 1999).

[2] O. Bottema, B. Roth, Theoretical Kinematics, North Holland Publishers, 1979, Dover Publishers, 1990.

[3] L. Brand, Vector and Tensor Analysis, John Wiley, 1947.

[4] V. Brodsky, M. Shoham, Dual numbers representation of rigid body dynamics, Mechanism and Machine Theory 34 (1999) 693718.

[5] N. Ciblak, H. Lipkin, Asymmetric Cartesian stiffness for the modelling compliant robotic systems, ASME Design Technical Conferences, DE vol. 72, September 11 14, 1994, pp. 197204.

[6] F.M. Dimentberg, The Screw Calculus and Its Applications in Mechanics, Foreign Technology Division translation, FTD HT 1632 67, 1965.

[7] R. Featherstone, Robot Dynamics Algorithms, Kluwer Academic Publishers, 1987.

[8] R. Featherstone, The acceleration vector of a rigid body, International Journal of Robotics Research 20 (11) (2001) 841846

[9] M. Griffis, J. Duffy, Global stiffness modeling of a class of simple compliant couplings, Mechanism and Machine Theory 28 (2) (1993).

[10] W.S. Howard, M. Zefran, V. Kumar, On the $6 \times 6$ Cartesian stiffness matrix for three dimensional motions, Mechanism and Machine Theory 33 (4) (1998).

[11] K.H. Hunt, Kinematic Geometry of Mechanisms, Oxford Press, 1978.

[12] A. Karger, J. Novak, Space Kinematics and Lie Groups, Gordon and Breach Science Publishers, New York, 1985.

[13] H. Lipkin, Extension of Euler's principal axes to articulated inertia, in: Proceedings of the Ninth World Congress on the Theory of Machines and Mechanisms, Milano, August 29 September 2, 1995, vol. 2, pp. 14751479.

[14] H. Lipkin, J. Duffy (Eds.), Proceedings of a Symposium Commemorating the Legacy, Works, and Life of Sir Robert Stawell Ball Upon the 100th Anniversary of A Treatise on the Theory of Screws, July 9 11, 2000, University of Cambridge, Trinity College. Available from: <http://helix. gatech. edu/ball2000>.

[15] G.H. Lovell, A New Approach to Motor Calculus and Rigid Body Dynamics with Applications to Serial Open Loop Chains, Ph.D. Dissertation, University of Florida, 1986.

[16] L.E. Malvern, Introduction to the Mechanics of a Continuous Medium, Prentice Hall, NJ, 1969.

[17] G.R. Pennock, P.J. Meehan, Geometric insight into the dynamics of a rigid body using the theory of screws, in: Proceedings of a Symposium Commemorating the Legacy, Works, and Life of Sir Robert Stawell Ball Upon the 100th Anniversary of A Treatise on the Theory of Screws, July 9 11, 2000, University of Cambridge, Trinity College. Available from: <http://helix. gatech. edu/ball2000>.

[18] J. Phillips, Introducing Screw Theory/Screw Theory Exemplified, Freedom in Machinery, vol. 1/2, Cambridge University Press, 1984/1990. 
[19] T. Pigoski, M. Griffis, J. Duffy, Stiffness mappings employing different frames of reference, in: Proceedings 22nd Biennial ASME Mechanisms Conference, September 1992, Phoenix, DE vol. 47, pp. 445452.

[20] J.M. Rico, J. Duffy, An application of screw algebra to the acceleration analysis of serial chains, Mechanism and Machine Theory 31 (4) (1996).

[21] J.M. Rico, J. Gallardo, J. Duffy, Screw theory and higher order kinematic analysis of open serial and closed chains, Mechanism and Machine Theory 34 (4) (1999) 559586.

[22] P. Ridley, E.H. Bokelberg, K.H. Hunt, Spatial motion I \& II, Mechanism and Machine Theory 27 (1) (1992) 135.

[23] J.M. Selig, Geometrical Methods in Robotics, Springer, New York, 1994.

[24] J.M. Selig, Three problems in robotics, Journal of Mechanical Engineering Science, part c 216 (2002) 7180.

[25] S. Stramigioli, H. Bruynickx, Geometry of dynamic and higher order kinematic screws, in: Proceedings IEEE Robotic and Automation Conference, Seoul, Korea, May 21 26, 2001.

[26] M.M. Svinin, S. Hosoe, M. Uchiyama, On the stiffness and stability of Gough Stewart platforms, in: Proceedings IEEE Robotic and Automation Conference, Seoul, Korea, May 21 26, 2001.

[27] C.R. Tischler, D.M. Downing, S.R. Lucas, D. Martins, Rigid body inertia and screw geometry, in: Proceedings of a Symposium Commemorating the Legacy, Works, and Life of Sir Robert Stawell Ball Upon the 100th Anniversary of A Treatise on the Theory of Screws, July 9 11, 2000, University of Cambridge, Trinity College. Available from: <http://helix. gatech. edu/ball2000>.

[28] R. von Mises, Motorrechnung ein neues Hilfsmittel der Mechanik, Zeitschrift für Angewandte Mathematik und Mechanik 4 (2) (1924) 124 155, Motor Calculus, A New Theoretical Device for Mechanics, English translation by E.J. Baker, K. Wohlhart, Institute for Mechanics, University of Graz, 1996.

[29] K. Wohlhart, Motor tensor calculus, in: J. P. Merlet, B. Ravani (Eds.), Computational Kinematics, Kluwer Academic Pub., 1995, pp. 93102.

[30] A.T. Yang, Acceleration analysis of spatial four link mechanism, ASME Journal of Engineering for Industry, Series B 88 (2) (1966) 296300.

[31] A.T. Yang, Inertia force analysis of spatial mechanisms, ASME Journal of Engineering for Industry (1971) 2733. 